\title{
Combined effects of furanodiene and doxorubicin on the migration and invasion of MDA-MB-231 breast cancer cells in vitro
}

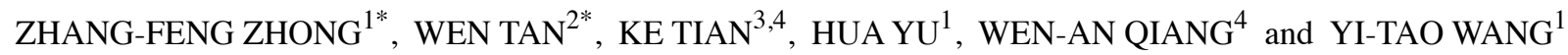 \\ ${ }^{1}$ Institute of Chinese Medical Sciences, State Key Laboratory of Quality Research in Chinese Medicine, \\ University of Macau, Macao SAR 999078; ${ }^{2}$ School of Pharmacy, Lanzhou University, Lanzhou, Gansu 730000; \\ ${ }^{3}$ School of Chinese Medicine, Hong Kong Baptist University, Hong Kong SAR 999077, P.R. China; \\ ${ }^{4}$ Division of Reproductive Science in Medicine, Department of Obstetrics and Gynecology, \\ Feinberg School of Medicine at Northwestern University, Chicago, IL 60611, USA
}

Received February 19, 2016; Accepted March 11, 2016

DOI: $10.3892 / o r .2017 .5435$

\begin{abstract}
Furanodiene is one of the major bioactive components isolated from the natural product of the plant, Curcuma wenyujin Y.H. Chen et C. Ling. Furanodiene has been found to exert anticancer effects in various types of cancer cell lines, as well as exhibit antimetastatic activities. However, the antimetastatic capacity of furanodiene in combination with the common chemotherapy drug doxorubicin has not been investigated. We found that doxorubicin at a non-toxic concentration induced cell migration and cell invasion in highly metastatic breast cancer cells. Combinational treatments with furanodiene and doxorubicin blocked the invasion and migration of MDA-MB-231 breast
\end{abstract}

Correspondence to: Professor Yi-Tao Wang, Institute of Chinese Medical Sciences, University of Macau, Avenida da Universidade, Taipa, Macao SAR 999078, P.R. China

E-mail: ytwang@umac.mo

Dr Wen-An Qiang, Department of Obstetrics and Gynecology, Feinberg School of Medicine at Northwestern University, 300 East Superior Street, Lurie 7-250, Chicago, IL 60611, USA

E-mail: w-qiang@northwestern.edu

*Contributed equally

Abbreviations: Dox, doxorubicin; Fur, furanodiene; MMP-9, matrix metalloproteinase-9; TGF- $\beta$, transforming growth factor- $\beta$; PI3K, phosphoinositide 3-kinase; FBS, fetal bovine serum; PBS, phosphatebuffered saline; PS, penicillin-streptomycin; PI, propidium iodide; TPA, tetradecanoylphorbol acetate; DMSO, dimethyl sulfoxide; RIPA, radioimmunoprecipitation assay; SDS, sodium dodecyl sulfate; PAGE, polyacrylamide gel electrophoresis; PVDF, polyvinylidene fluoride; PFA, paraformaldehyde

Key words: furanodiene, doxorubicin, combination treatment, breast cancer, migration, invasion cancer cells in vitro. We also clarified the effects of the combination on the signaling pathways involved in migration, invasion, and cytoskeletal organization. When combined with doxorubicin, furanodiene downregulated the expression of integrin $\alpha \mathrm{V}$ and $\beta$-catenin and inhibited the phosphorylation of paxillin, Src, focal adhesion kinase (FAK), p85, and Akt. Moreover, combinational treatments also resulted in a decrease in matrix metalloproteinase-9 (MMP-9). Further study demonstrated that the co-treatments with furanodiene did not significantly alter the effects of doxorubicin on the tubulin cytoskeleton, represented by no influence on the expression levels of RhoA, Cdc 42 , N-WASP, and $\alpha / \beta$ tubulin. These observations indicate that furanodiene is a potential agent that may be utilized to improve the anticancer efficacy of doxorubicin and overcome the risk of chemotherapy in highly metastatic breast cancer.

\section{Introduction}

Chemotherapy is an effective approach by which to treat various types of cancer. However, chemotherapy regimens also carry the risks of side-effects, drug resistance, and serious complications (1-3). Thus, adjuvant chemotherapy is a rational way to overcome the side-effects $(4,5)$. Numerous natural products have been associated with cancer prevention and treatment, and the bioactive compounds derived from natural products are always applied in combination with chemotherapy drugs (6-8). Doxorubicin is a first-line chemotherapy drug for the treatment of breast cancer, hepatocellular carcinoma and gastric cancer. In addition to its therapeutic activities, doxorubicin also enhances the malignant properties of cancer cells in clinical chemotherapy. Recent studies claim that doxorubicin induces serious side-effects, such as cardiomyopathy (9) and metastatic potential (10). Numerous studies have shown that many bioactive compounds have the capability to improve the anticancer activity of doxorubicin and reduce its side-effects $(11,12)$. However, there have been few studies focusing on the metastatic potential induced by doxorubicin in chemotherapy. Therefore, it is significant to 
explore effective compounds derived from natural products to reverse the metastatic potential induced by doxorubicin.

Curcuma wenyujin Y.H. Chen et C. Ling is a commonly used Chinese herb, which contains many bioactive components such as curcumin, $\beta$-elemene, and furanodiene $(13,14)$. Curcumin exhibits antimetastatic properties via reducing the expression levels of $\alpha 5 \beta 1$ and $\alpha 5 \beta 3$ integrin and inhibiting the phosphorylation of focal adhesion kinase (FAK), Src, and PI3K/Akt (15-17). In adjuvant cancer treatment, curcumin suppressed the epithelial-mesenchymal transition induced by doxorubicin via the regulation of TGF- $\beta$ and PI3K/Akt signaling pathways in triple-negative breast cancer cells (18). In addition, a previous study reported that there were no significant changes induced by curcumin in the Wnt/ $\beta$-catenin pathway (19). $\beta$-elemene, another well-known bioactive component, was also found to exhibit antimetastatic properties in melanoma and breast cancer in vitro or in vivo $(20,21)$. Growing evidence shows that furanodiene exerts pro-apoptotic, anti-angiogenic, and antimetastatic effects in several cancer cell lines $(22,23)$. Furanodiene inhibited the adhesion, migration, and invasion of breast cancer cells through the regulation of integrin $\alpha \mathrm{V}$, $\beta$-catenin, FAK, PI3K/Akt, and MMP signaling pathways (24). However, the antimetastatic capacity of furanodiene in combination with the common chemotherapy drug doxorubicin has not been well investigated. Therefore, we initially studied the combined effects of furanodiene and doxorubicin against cancer cell migration and invasion with the view of improving treatment efficacy and overcoming side-effects.

\section{Materials and methods}

Chemicals and reagents. Furanodiene was purchased from the National Institutes for Food and Drug Control (Beijing, China). RPMI-1640 culture medium was purchased from Gibco (Rockville, MD, USA). Fetal bovine serum (FBS), phosphatebuffered saline (PBS), penicillin-streptomycin (PS), Tubulin Tracker Green, 3-[4,5-dimethyl-2-thiazolyl]-2,5-diphenyltetrazolium bromide (MTT), propidium iodide (PI), and $0.25 \%$ (w/v) trypsin/1 mM EDTA were obtained from Invitrogen (Carlsbad, CA, USA). Doxorubicin and tetradecanoylphorbol acetate (TPA) were supplied by Sigma-Aldrich (St. Louis, MO, USA). Radioimmunoprecipitation assay (RIPA) lysis buffer was obtained from Santa Cruz Biotechnology (Santa Cruz, CA, USA). Primary antibodies against caspase 8 , Bcl-2, Bax, caspase 7, PARP, integrin $\alpha \mathrm{V}, \beta$-catenin, RhoA, Cdc42, N-WASP, $\alpha / \beta$ tubulin, Akt, p-Akt (Ser473), p-paxillin (Tyr118), p-Src (Tyr416), p-FAK (Tyr925), p-p85 (Ser428), and GAPDH were purchased from Cell Signaling Technology (Danvers, MA, USA), as well as the secondary antibodies. Matrigel and Transwell chamber systems were obtained from BD Biosciences (Bedford, MA, USA). MMP-9 immunoassay kit was purchased from R\&D Systems (Minneapolis, MN, USA).

Cell culture and drug treatment. MDA-MB-231 cell line was obtained from the American Type Culture Collection (ATCC; Manassas, VA, USA) and cultured as previously reported (25). The stock solution of furanodiene $(20 \mathrm{mM})$ and doxorubicin $(100 \mu \mathrm{M})$ dissolved in dimethyl sulfoxide (DMSO) was diluted to different concentrations as designed.
Cell viability assay. Cell viability was assessed by MTT assay as previously described (26). Briefly, exponentially growing MDA-MB-231 cells $\left(1 \times 10^{4}\right)$ in $100 \mu 1$ were seeded into 96-well tissue culture plates. Cells were treated with different concentrations of furanodiene $(0,5,10$ and $20 \mu \mathrm{M})$ in the presence or absence of doxorubicin $(0.1 \mu \mathrm{M})$ for $12 \mathrm{~h}$. The cell viability was determined by adding $100 \mu \mathrm{l}$ of MTT $(1 \mathrm{mg} / \mathrm{ml})$. Following an incubation period $(4 \mathrm{~h})$, the absorbance values at $570 \mathrm{~nm}$ were recorded using a Multi-label Counter (PerkinElmer, Singapore).

Western blotting assay. Western blotting assay was performed according to a previous study (23). Briefly, MDA-MB-231 cells $\left(1 \times 10^{6}\right)$ were seeded in $60-\mathrm{mm}$ Petri dish and were treated with different concentrations of furanodiene $(0,5,10$ and $20 \mu \mathrm{M})$ in the presence and absence of doxorubicin $(0.1 \mu \mathrm{M})$. The cells were harvested after a 12-h treatment, and the total proteins were extracted with RIPA lysis buffer. Equal amounts of total protein were separated by appropriate SDS-PAGE followed by transferring onto a polyvinylidene fluoride (PVDF) membrane. Following blocking with non-fat milk for $1 \mathrm{~h}$, the membrane was incubated with a specific primary antibody $(1: 1,000)$ for $2 \mathrm{~h}$ and the corresponding secondary antibody $(1: 1,000)$ for $1 \mathrm{~h}$, respectively. The specific protein bands were visualized using an ECL Advanced Western Blotting Detection kit (Amersham Life Sciences, UK).

Cell cycle assay. Cell cycle distribution was determined according to a previous study (27). Briefly, MDA-MB-231 cells $\left(2 \times 10^{5}\right)$ were seeded into a 6 -well plate and were treated with different concentrations of furanodiene $(0,5,10$ and $20 \mu \mathrm{M})$ in the presence and absence of doxorubicin $(0.1 \mu \mathrm{M})$ for $12 \mathrm{~h}$. Then, the cells were collected and fixed with ice-cold $70 \%$ ethanol at $-20^{\circ} \mathrm{C}$ overnight. The cells were procured again by centrifuging and were incubated with PI staining solution for 30 min protected from light. The cell cycle distribution was analyzed using flow cytometry (BD FACSCanto ${ }^{\mathrm{TM}}$; BD Biosciences, San Jose, CA, USA). The results were analyzed by ModFit LT software (version 3.0).

Transwell invasion assay. The invasive potential of the cells was assessed by a three-dimensional (3-D) cell invasion assay as previously described (28). Briefly, the upper and lower sides of the membrane were pre-coated with 1:30 (v/v) and 1:100 (v/v) of Matrigel, respectively. Cell density was adjusted to $1 \times 10^{5} / \mathrm{ml}$. The cell suspension $(500 \mathrm{ml})$ containing serum-free RPMI-1640 medium and different concentrations of furanodiene $(0,0.1$ and $20 \mu \mathrm{M})$ and doxorubicin $(0.1 \mu \mathrm{M})$ (alone or in combination) was deposited into the upper chamber of each well. The lower chambers were loaded with RPMI-1640 medium containing $1 \% \mathrm{FBS}(\mathrm{v} / \mathrm{v}$, as a chemoattractant), different concentrations of furanodiene $(0,0.1$ and $20 \mu \mathrm{M})$, and doxorubicin $(0.1 \mu \mathrm{M})$ (alone or in combination). After a 12-h incubation, the noninvasive MDA-MB-231 cells were removed by gentle scraping. The invasive cells were fixed with paraformaldehyde (PFA; $3.7 \% / \mathrm{v} / \mathrm{v}$ ) at room temperature for $30 \mathrm{~min}$, and then stained with Hoechst 33342 for $15 \mathrm{~min}$. Images of invasive cells were captured at a magnification of x100 using a digital camera. The number of invasive cells was counted using a MetaMorph Imaging Series software (Molecular Devices, Tokyo, Japan). 
Transwell migration assay. MDA-MB-231 cells were treated with furanodiene $(0$ and $20 \mu \mathrm{M})$ in the presence or absence of doxorubicin $(0.1 \mu \mathrm{M})$ for $12 \mathrm{~h}$. Cell migration assay was performed as described in the previous section (Transwell invasion assay) with a slight modification in that both sides of the insert were not pre-coated with Matrigel.

Real-time migration assay. Real-time cell migration was continuously determined by the xCELLigence system (Roche, Mannheim, Germany) as previously described (24). The density of the cell suspension was adjusted to $2 \times 10^{5}$ cells $/ \mathrm{ml}$. The upper chamber of each well was filled with $100 \mu \mathrm{l}$ of cell suspension containing serum-free RPMI-1640 medium and different concentrations of furanodiene $(0,5,10$ and $20 \mu \mathrm{M})$ and doxorubicin $(0.1 \mu \mathrm{M})$ (alone or in combination). The lower chambers were loaded with RPMI-1640 medium containing $1 \% \mathrm{FBS}(\mathrm{v} / \mathrm{v}$, as a chemoattractant), corresponding concentrations of furanodiene $(0,5,10$ and $20 \mu \mathrm{M})$, and doxorubicin $(0.1 \mu \mathrm{M})$ (alone or in combination). Firstly, the CIM-Plate was placed in an incubator to allow cell sedimentation for $30 \mathrm{~min}$. Then, the RTCA DP analyzer started to record in an incubator, and the duration of measurement was $12 \mathrm{~h}$ with an interval of 5 min.

Gelatin zymography assay. A gelatin zymography assay was performed according to a previous study (24). In brief, MDA-MB-231 cells $\left(2 \times 10^{5}\right)$ were seeded into a 6-well plate, and treated with different concentrations of furanodiene $(0,5$, 10 and $20 \mu \mathrm{M})$ in the presence and absence of doxorubicin $(0.1 \mu \mathrm{M})$ in RPMI-1640 medium (containing 1\% FBS and $20 \mathrm{ng} / \mathrm{ml} \mathrm{TPA}$ ) for $12 \mathrm{~h}$. The culture medium was centrifuged at $350 \mathrm{x} \mathrm{g}$ for $5 \mathrm{~min}$ at $4^{\circ} \mathrm{C}$. In addition, $20 \mu \mathrm{l}$ of the supernatant was mixed with $5 \mu 1$ of the non-reducing sample buffer [5X, $62.5 \mathrm{mM}$ Tris-HCl, $\mathrm{pH} \mathrm{6.8,2 \%} \mathrm{(v/v)} \mathrm{SDS,} \mathrm{25 \%} \mathrm{(v/v)} \mathrm{glycerol,}$ $0.01 \%$ (v/v) bromophenol blue]. Then, the mixed sample $(20 \mu \mathrm{l})$ was separated on a $7 \%$ polyacrylamide gel $(0.1 \%$ gelatin) at $100 \mathrm{~V}$ for $75 \mathrm{~min}$ and was rinsed with renaturing buffer for $1.5 \mathrm{~h}$ at room temperature. After a $42-\mathrm{h}$ incubation in developing buffer at $37^{\circ} \mathrm{C}$, the gel was stained with Coomassie blue and was destained with $15 \%$ acetic acid.

Quantitative immunoassay for matrix metalloproteinase- 9 (MMP-9). Quantitative immunoassay for MMP-9 was performed according to a previous study (29). MDA-MB-231 cells $\left(5 \times 10^{4}\right)$ were seeded in each well of 24 -well plates. The cells were treated with furanodiene $(20 \mu \mathrm{M})$ and doxorubicin $(0.1 \mu \mathrm{M})$ (alone or in combination) in the presence or absence of TPA $(20 \mathrm{ng} / \mathrm{ml})$ for $12 \mathrm{~h}$. The conditioned culture medium was collected and the level of MMP-9 secreted from the cells was determined using immunoassay kit (R\&D Systems, Minneapolis, MN, USA) according to the manufacturer's protocol. The absorbance values at 450 and $570 \mathrm{~nm}$ were recorded using a Multi-label Counter (PerkinElmer).

Tubulin level assay. MDA-MB-231 cells $\left(2 \times 10^{5}\right)$ were seeded into 6-well tissue culture plates and were incubated in a humidified incubator for $24 \mathrm{~h}$. The cells were treated with different concentrations of furanodiene $(0,5,10$ and $20 \mu \mathrm{M})$ in the presence and absence of doxorubicin $(0.1 \mu \mathrm{M})$ for $12 \mathrm{~h}$. The induced cells were harvested and washed with PBS, and were then re-suspended in serum-free RPMI-1640 medium containing $100 \mathrm{nM}$ Tubulin Tracker Green probe for a further 30 -min incubation at $37^{\circ} \mathrm{C}$. For quantitative assessment, the cells were collected and re-suspended in PBS, followed by analysis via flow cytometry (BD FACSCanto ${ }^{\mathrm{TM}}$ ). The fluorescence of 10,000 analyzed cells (corrected for autofluorescence) in each group represents the tubulin level.

Data analysis. All data represent the mean of 3 separately performed experiments and were represented as mean \pm SEM. The significance of group differences was evaluated via one-way ANOVA using GraphPad Prism software (GraphPad Software, Inc., San Diego, CA, USA). Newman-Keuls multiple comparison tests were performed for post hoc pairwise comparisons. Statistical differences were considered significant at $\mathrm{P}<0.05$. Data from at least 3 independent experiments were used for the statistical analysis.

\section{Results}

Co-treatments with furanodiene $(0-20 \mu \mathrm{M})$ do not enhance the cytotoxic effect of doxorubicin $(0.1 \mu M)$ in MDA-MB-231 cells. The cytotoxic effects of furanodiene and doxorubicin in the highly metastatic MDA-MB-231 human breast cancer cells were determined by MTT assay. As shown in Fig. 1A, co-treatments with furanodiene $(0-20 \mu \mathrm{M})$ did not enhance the cytotoxicity of doxorubicin $(0.1 \mu \mathrm{M})$. Accordingly, we used different methods to confirm cell status alteration after co-treatments with furanodiene $(0-20 \mu \mathrm{M})$ and doxorubicin $(0.1 \mu \mathrm{M})$. Our results showed that co-treatments with furanodiene $(0-20 \mu \mathrm{M})$ and doxorubicin $(0.1 \mu \mathrm{M})$ did not induce the cleavage of caspase 8 and 7, and PARP and also did not affect the expressions of Bcl-2 and Bax (Fig. 1B). These results confirmed that the co-treatments did not induce apoptosis in the MDA-MB-231 cells. Furthermore, the co-treatments did not induce cell cycle arrest at the corresponding concentrations, indicating that the cell status was normal after the co-treatments with furanodiene $(0-20 \mu \mathrm{M})$ and doxorubicin $(0.1 \mu \mathrm{M})$ for $12 \mathrm{~h}$ in the MDA-MB-231 cells (Fig. 1C).

Combined effects of furanodiene and doxorubicin on cell invasion and migration in MDA-MB-231 cells. Doxorubicin $(0.1 \mu \mathrm{M})$ treatment alone induced a significant increase in invasion (Fig. 2A) and migration (Fig. 2B) of the MDA-MB-231 cells, with 1.36- and 3.92-fold, respectively. Furanodiene $(0.1 \mu \mathrm{M})$ treatment alone did not significantly affect the cell invasion, but markedly attenuated the invasion of the MDA-MB-231 cells induced by doxorubicin (Fig. 2A). Furthermore, co-treatments with furanodiene $(20 \mu \mathrm{M})$ and doxorubicin $(0.1 \mu \mathrm{M})$ resulted in much more marked reduction in the invasion (Fig. 2A) and the migration (Fig. 2B) of MDA-MB-231 cells than that of furanodiene $(20 \mu \mathrm{M})$ treatment alone. Fig. $2 \mathrm{C}$ and $\mathrm{D}$ are statistical results of the cell invasion and migration.

Meanwhile, our real-time results also demonstrated an increase in the migration of MDA-MB-231 cells following doxorubicin $(0.1 \mu \mathrm{M})$ incubation, and furanodiene $(0-20 \mu \mathrm{M})$ treatment attenuated the doxorubicin-induced migration gradually, even at the low concentration of $5 \mu \mathrm{M}$ within 12 h (Fig. 3). 
A

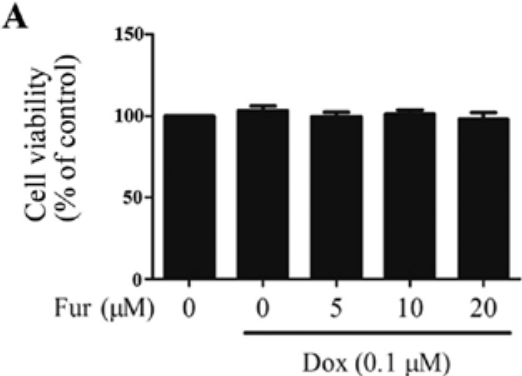

B

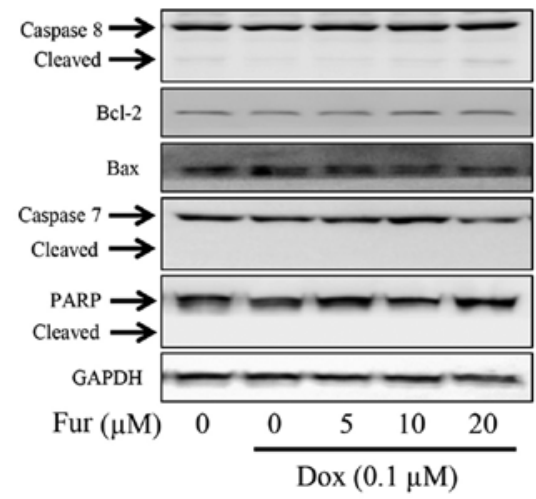

C

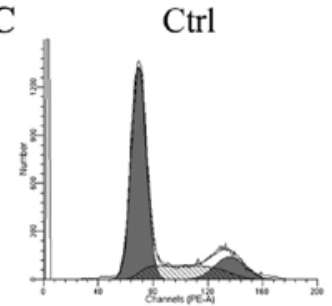

Fur5 + Dox

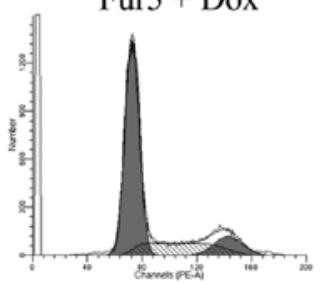

Fur20 + Dox

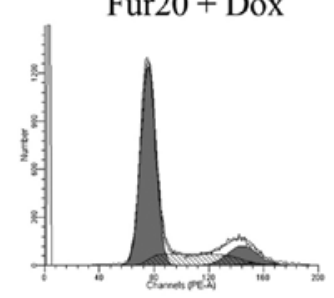

Dox

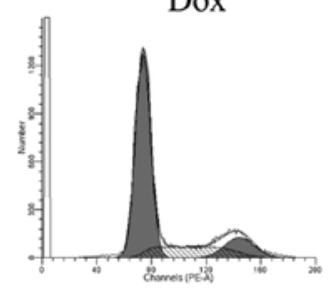

Fur10 + Dox
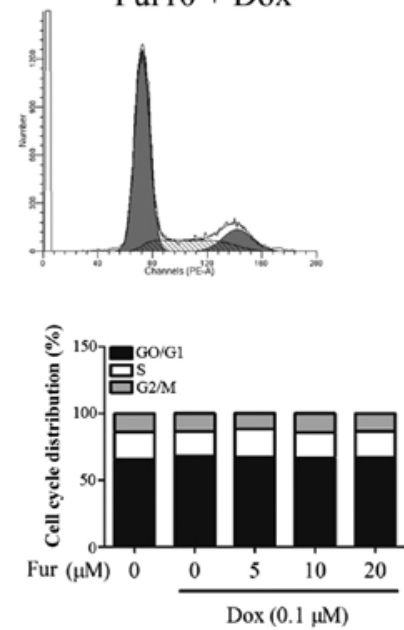

Figure 1. Combined effects of doxorubicin (Dox) and furanodiene (Fur) on cell viability, cell apoptosis and cell cycle distribution in MDA-MB-231 cells Cells were co-treated with Dox $(0.1 \mu \mathrm{M})$ and Fur $(0,5,10$ and $20 \mu \mathrm{M})$ for $12 \mathrm{~h}$. (A) Cell viability was detected by MTT assay. (B) Biomarkers of cell apoptosis were detected by western blotting. (C) Cell cycle was analyzed by flow cytometry. Histograms represent the cell cycle distribution and the ratios were also calculated. Ctrl, control.

Combined effects of furanodiene and doxorubicin on the metastasis-related proteins in MDA-MB-231 cells. To explore the underlying mechanisms involved in the antimetastatic effects of furanodiene and doxorubicin in the MDA-MB-231 cells, the expression levels of several proteins related to the regulation of metastasis were determined by western blotting. Our results showed that doxorubicin $(0.1 \mu \mathrm{M})$ treatment alone increased the expression of integrin $\alpha \mathrm{V}$ and induced the phosphorylation of FAK, Src, paxillin, p85 and Akt. However, co-treatments with furanodiene (5-20 $\mu \mathrm{M})$ and doxorubicin $(0.1 \mu \mathrm{M})$ downregulated the expression levels of integrin $\alpha \mathrm{V}$ and $\beta$-catenin and inhibited the phosphorylation of FAK, Src, paxillin, p85, and Akt (Fig. 4A).

Co-treatments with furanodiene and doxorubicin attenuate the MMP-9 release induced by TPA in MDA-MB-231 cells. The gelatin enzymography assay was performed to investigate whether co-treatments with furanodiene and doxorubicin suppress breast cancer cell invasion by affecting the activity of MMP-9. Our results showed that co-treatment with furanodiene $(20 \mu \mathrm{M})$ and doxorubicin $(0.1 \mu \mathrm{M})$ markedly attenuated TPA-induced MMP-9 activity. However, lower concentrations of furanodiene (5 and $10 \mu \mathrm{M})$ did not significantly affect the MMP-9 activity induced by TPA $(20 \mathrm{ng} / \mathrm{ml})$ and doxorubicin $(0.1 \mu \mathrm{M})$ (Fig. 4B). Afterwards, the ELISA immunoassay was initially used to confirm the changes in MMP-9 release in the conditioned medium after co-treatment with furanodiene and doxorubicin. As shown in Fig. 4C, the MMP-9 release induced by TPA in the conditioned medium decreased to $50.50 \%$ with significance in response to co-treatment with furanodiene $(20 \mu \mathrm{M})$ and doxorubicin $(0.1 \mu \mathrm{M})$.

Combined effects of furanodiene and doxorubicin on cell cytoskeleton reorganization. To determine whether the cell cytoskeleton reorganization may be involved in the antimetastatic effects of furanodiene and doxorubicin, the cytoskeletal regulatory proteins were detected by western blotting. As shown in Fig. 5A, our results showed that co-treatments with furanodiene $(0-20 \mu \mathrm{M})$ and doxorubicin $(0.1 \mu \mathrm{M})$ did not alter the expression levels of RhoA, Cdc42, N-WASP, and $\alpha / \beta$ tubulin in the MDA-MB-231 cells. Furthermore, the tubulin level was also detected with Tubulin Tracker Green probe by flow cytometry. In addition, the results showed that tubulin assembly was not significantly regulated by co-treatments with furanodiene $(0-20 \mu \mathrm{M})$ and doxorubicin $(0.1 \mu \mathrm{M})$ for $12 \mathrm{~h}$ (Fig. 5B and C).

\section{Discussion}

Although chemotherapy is an effective approach to prolong survival and reduce mortality, the chemotherapy-induced sideeffects must not be overlooked (30-32). Combinational therapy with reduced drug dosages is commonly an effective approach to prevent side-effects. However, sometimes low dosage may increase the metastatic potential, which is related to cancer recurrence. Several first-line drugs, such as oxaliplatin, cyclophosphamide, and doxorubicin, were reported for conspicuous side-effects, namely chemotherapy-driven metastasis (33-35). 

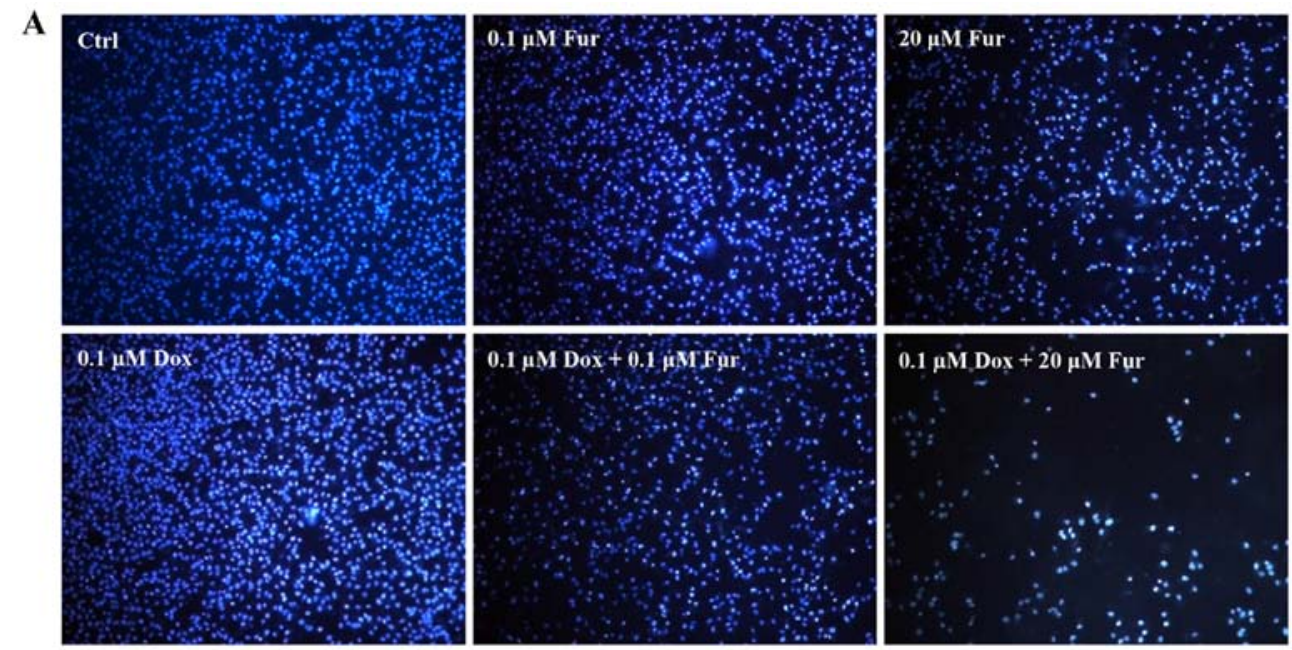

B
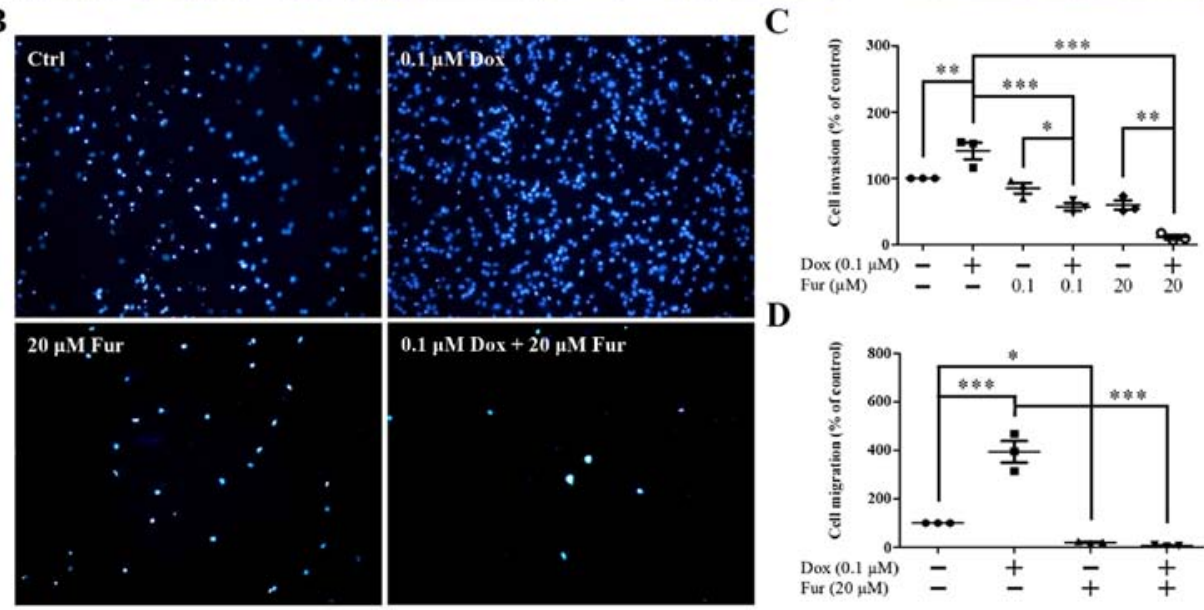

D

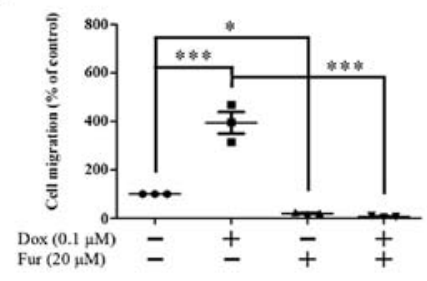

Figure 2. Combined effects of doxorubicin (Dox) and furanodiene (Fur) on MDA-MB-231 cell invasion and migration in a 3-D model. Cells were co-treated with doxorubicin $(0.1 \mu \mathrm{M})$ and furanodiene $(0,0.1$ and $20 \mu \mathrm{M})$ for $12 \mathrm{~h}$. (A) Furanodiene attenuated doxorubicin-induced MDA-MB-231 cell invasion compared with the negative control (Ctrl). (B) Furanodiene attenuated doxorubicin-induced MDA-MB-231 cell migration compared with the negative control (Ctrl). (C) Statistical result of A. (D) Statistical result of B $\left({ }^{*} \mathrm{P}<0.05,{ }^{* * *} \mathrm{P}<0.01\right.$, and $\left.{ }^{* * * *} \mathrm{P}<0.001\right)$.

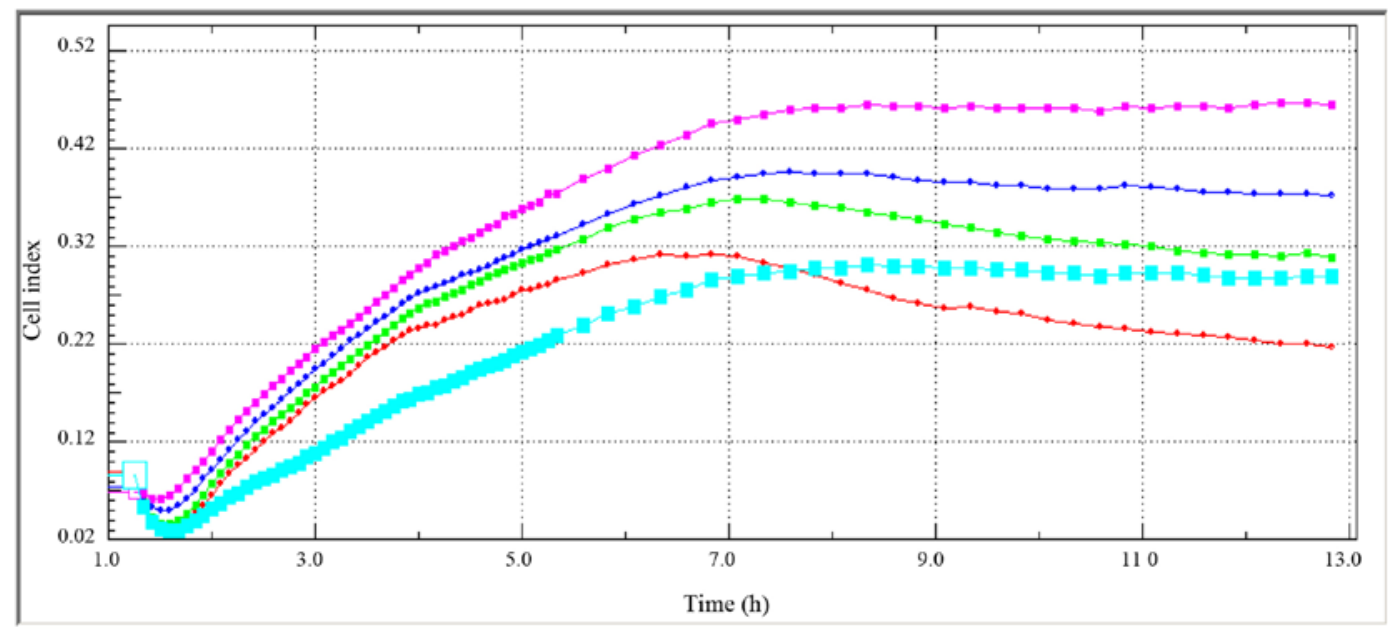

\begin{tabular}{|c|c|c|c|c|c|}
\hline & Light blue & Pink & Dark blue & Green & Red \\
\hline Dox $(\mu \mathrm{M})$ & 0 & 0.1 & 0.1 & 0.1 & 0.1 \\
\hline Fur $(\mu \mathrm{M})$ & 0 & 0 & 5 & 10 & 20 \\
\hline
\end{tabular}

Figure 3. Combined effects of doxorubicin (Dox) and furanodiene (Fur) on MDA-MB-231 cell migration in a real-time model. Real-time cell migration was continuously determined by the xCELLigence system and recorded with RTCA DP analyzer. The duration of measurement was $12 \mathrm{~h}$ with an interval of $5 \mathrm{~min}$. Furanodiene $(0-20 \mu \mathrm{M})$ attenuated doxorubicin $(0.1 \mu \mathrm{M})$-induced migration in the MDA-MB-231 cells. 
B

A

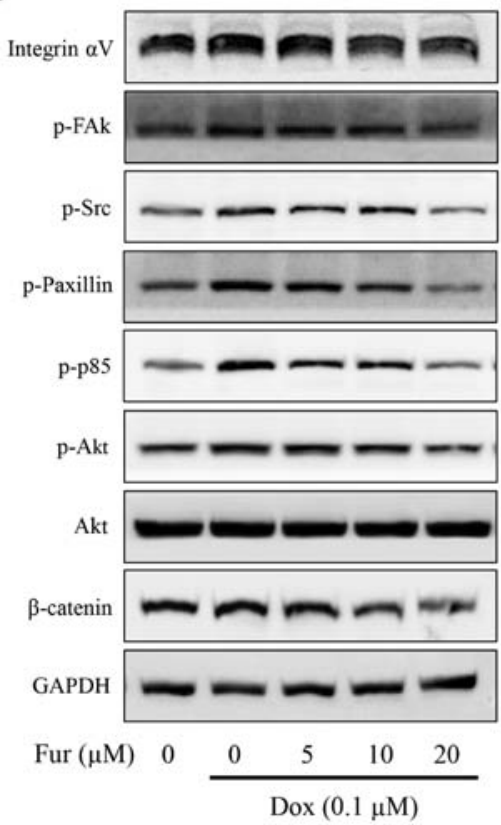

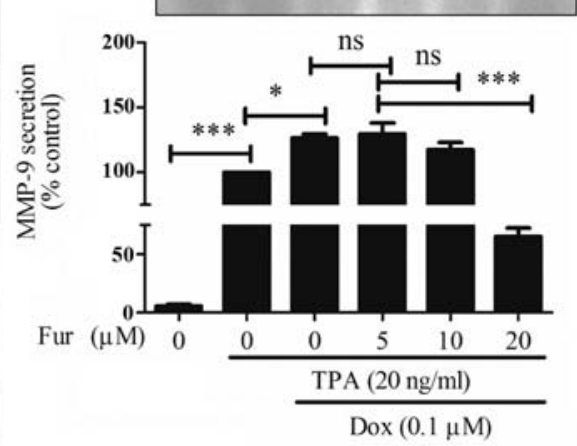

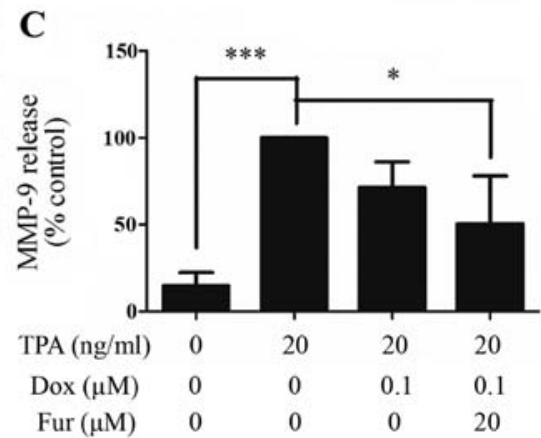

Figure 4. Combined effects of doxorubicin (Dox) and furanodiene (Fur) on the metastasis-associated pathways in MDA-MB-231 cells. Cells were co-treated with Dox $(0.1 \mu \mathrm{M})$ and Fur $(0-20 \mu \mathrm{M})$ for $12 \mathrm{~h}$, or in the presence of TPA $(20 \mathrm{ng} / \mathrm{ml})$. (A) The metastasis-associated proteins were detected by western blotting. (B) MMP-9 activity was analyzed by gelatin enzymography assay. (C) MMP-9 secretion level was detected using the ELISA kit ( $\mathrm{P}<0.05$ and $\left.{ }^{* * *} \mathrm{P}<0.001\right)$.

A

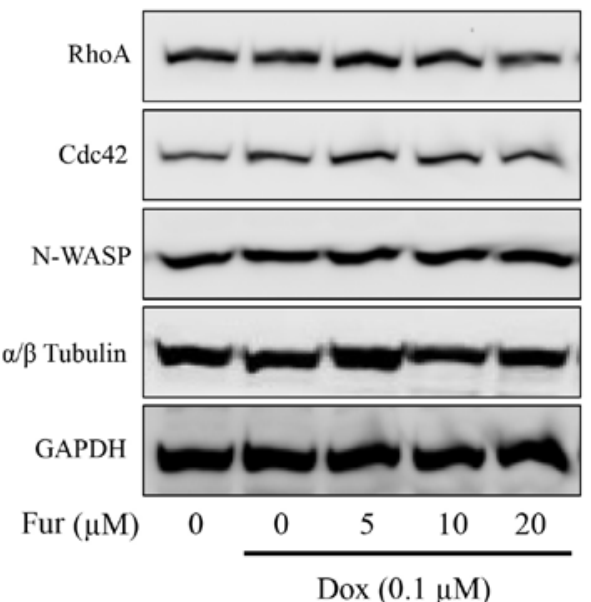

B

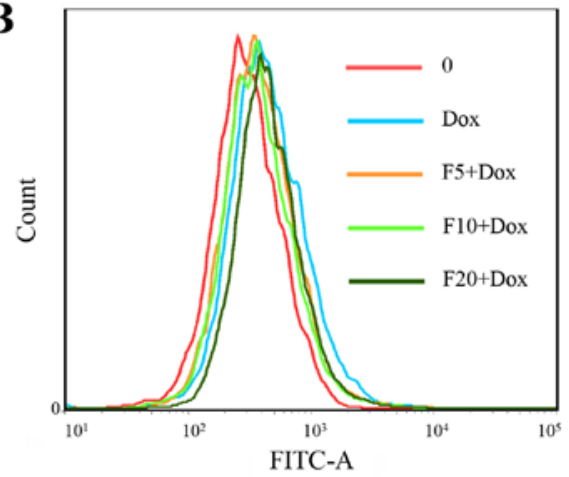

C

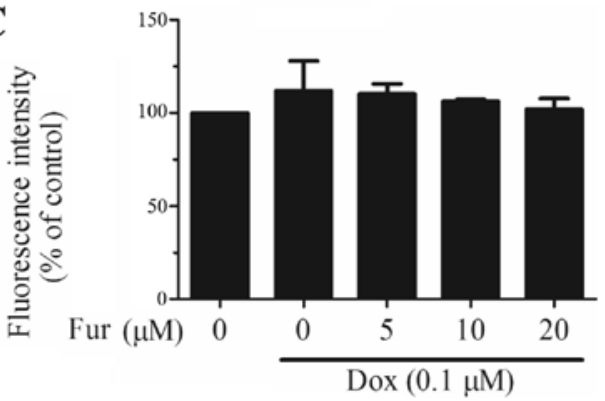

Figure 5. Combined effects of doxorubicin (Dox) and furanodiene (Fur or F) on cell cytoskeleton reorganization in the MDA-MB-231 cells. Cells were co-treated with doxorubicin $(0.1 \mu \mathrm{M})$ and furanodiene $(0-20 \mu \mathrm{M})$ for $12 \mathrm{~h}$. (A) The cytoskeleton reorganization associated proteins were detected by western blotting. (B) The cytoskeletal regulatory proteins were detected with Tubulin Tracker Green probe using flow cytometry. (C) Statistical results of B.

Metastasis is highlighted as one of the main problems $(34,36)$. Various studies have reported enhanced migration and invasion in MHCC97L and HepG2 cells in vitro and increased metastasis into the lung in hepatocellular carcinoma in vivo after oxaliplatin treatment (37). Cyclophosphamide was found to enhance pulmonary metastasis in T739 mice injected with 
LA795 cells and contribute to bone metastasis in a mouse model of prostate cancer (35). Likewise, doxorubicin administration increased the TGF- $\beta 1$ level and circulating tumor cells, thereby increasing lung metastasis in a MMTV/PyVmT transgenic model of metastatic breast cancer (38). In addition, doxorubicin treatment at certain dosages also enhanced metastasis to the lung in an MDA-MB-231 orthotopic xenograft model and metastasis to the bone in a $4 \mathrm{~T} 1$ orthotopic xenograft model $(10,39)$. In light of these studies, suppression of chemotherapy-driven metastasis is urgent and necessary.

As the major bioactive component isolated from Curcuma wenyujin Y.H. Chen et C. Ling, furanodiene exerts anticancer effects in various types of cancer cells as well as antimetastatic activities (24). Our previous study confirmed that furanodiene $(0-25 \mu \mathrm{M})$ treatment alone suppressed migration and invasion without inducing cytotoxicity in highly metastatic breast cancer cells (24). However, the antimetastatic capacity of furanodiene combined with the common chemotherapy drug doxorubicin has not yet been investigated. Our present study confirmed that doxorubicin $(0.1 \mu \mathrm{M})$ treatment caused a significant increase in the invasion and migration of highly metastatic breast cancer MDA-MB-231 cells. Expectably, furanodiene attenuated the invasion and the migration of MDA-MB-231 cells induced by doxorubicin even at nanomolar concentrations. Accordingly, furanodiene blocked doxorubicin-induced cell migration, which was indicated by real-time alterations of attachment and spreading of cells monitored by the XCELLigence system. Under the same conditions, this co-treatment with furanodiene did not enhance the cytotoxic effect of doxorubicin. Taking into account that no alteration in cell apoptosis and cell cycle distribution occurred following co-treatment, the attenuation of doxorubicin-induced migration and invasion by fuanodiene was independent of cell cytotoxicity. Collectively, the natural components extracted from Curcuma wenyujin Y.H. Chen et C. Ling may be potential natural products used to reduce the side-effects of chemotherapy agents and prevent cancer recurrence.

We investigated the underlying mechanisms involved in the antimetastatic effects of furandiene and doxorubicin in MDA-MB-231 cells. Integrins are $\alpha / \beta$ heterodimeric cell surface receptors that may be involved in embryonic development, angiogenesis, and tumor metastasis (40). Focal adhesion kinase (FAK) plays a pivotal role in the control of cell survival, spreading, and migration, which is in response to integrin-mediated signal transduction (41). Clustering of integrins leads to FAK activation and the autophosphorylation of FAK at Tyr397. This relationship between integrins and FAK gives rise to a series of subsequent events, including activation of Src family kinases, regulation of adhesion disassembly through paxillin, and activation of PI3K through $\mathrm{p} 85$ binding $(42,43)$. Additionally, PI3K activates the Akt cascade, which controls cell migration during the occurrence and development of cancer (44). In addition, the interaction of PI3K with $\beta$-catenin is necessary for the mediation of cell motility functions (45). All the proteins mentioned above compose signaling networks with antimetastatic effects, and furanodiene was found to interfere with these signaling networks to attenuate doxorubicin-induced migration and invasion. More specifically, doxorubicin-induced metastatic potential was confirmed by increased expression of integrin $\alpha \mathrm{V}$ and phosphorylation of
FAK, Src, paxillin, p85, and Akt after doxorubicin treatment alone. Afterwards, co-treatment with furanodiene and doxorubicin significantly reversed these increases in integrin $\alpha \mathrm{V}$ and the phosphorylation of FAK. Both are a central part in the downstream signaling regulation of cell motility and cell adhesion, as mentioned above. In addition to the suppression of the central part, marked inhibition of downstream signaling was also observed, including inhibition in the phosphorylation of Src, paxillin, p85, and Akt. Notably, Akt phosphorylation and $\beta$-catenin expression, which are associated with PI3K and implicated in cell migration and cell motility, were also decreased after the co-treatment with furanodiene and doxorubicin. Therefore, furanodiene interfered with the signaling networks to improve doxorubicin-induced migration and invasion in the MDA-MB-231 cells.

Matrix metalloproteinase (MMP) activities are associated with cancer occurrence and development. Among the family members, MMP-9 is an important factor during tumor invasion (46). TPA promotes MMP-9 activity by regulating the integrin pathway and proteolytic cleavage in human breast cancer cells (47). Co-treatment with furanodiene and doxorubicin markedly attenuated the TPA-induced MMP-9 activity in the highly metastatic breast cancer MDA-MB-231 cells.

Rho GTPases, including Rac1, RhoA and Cdc42, play a critical role in the regulation of actomyosin contractility and focal adhesion assembly (48). High expressions of RhoA and $\mathrm{Cdc} 42$ are regarded as adhesion markers (49). As a downstream effector, N-Wiskott-Aldrich (N-WASP; syndrome protein) serves an essential role in many actin-based processes, including the regulation of filopodium formation and actin polymerization (50). N-WASP binding to the Arp2/3 complex is required for Cdc42-dependent actin polymerization in reorganization of the actin cytoskeleton (51). In addition, this reorganization of the actin cytoskeleton is the primary mechanism for cell motility and migration (52). Inhibition of Rho GTPases and N-WASP leads to suppression of cell cytoskeleton reorganization. However, in the present study, no alterations were observed after co-treatment with furanodiene and doxorubicin. This indicated that the antimetastatic effects of furanodiene and doxorubicin may be independent of cell cytoskeleton reorganization. Additionally, there are 3 types of cytosolic fibers in the cytoskeleton, including microtubules, actin and intermediate filaments (53). Many microtubule-targeting drugs bind to $\alpha / \beta$ tubulin and disrupt microtubule dynamics to block mitosis in cancer cells. Likewise, no alteration in $\alpha / \beta$ tubulin was observed after co-treatment of furanodiene and doxorubicin in the MDA-MB-231 cells, including protein expression and florescence levels. Thus, the effects of the co-treatment with furanodiene and doxorubicin may be independent of cytoskeleton reorganization.

In conclusion, furanodiene attenuated the metastatic capacity of highly metastatic breast cancer cells induced by doxorubicin through the regulation of integrin $\alpha \mathrm{V}, \mathrm{FAK} /$ Src/paxillin, PI3K/Akt, $\beta$-catenin, and MMP-9 signaling pathways. In addition, the effects were independent of cytotoxicity and cell cytoskeleton reorganization. These results also indicate that natural products derived from Curcuma wenyujin Y.H. Chen et C. Ling may be developed as agents to reduce the side-effects caused by chemotherapeutic agents and prevent cancer recurrence. 


\section{Acknowledgements}

The present study was supported by the Macao Science and Technology Development Fund (077/2011/A3 and 048/2013/A2), the Research Fund of the University of Macau [CPG2014-00012-ICMS, UL016/09Y4/CMS/WYT01/ICMS and MYRG208 (Y3-L4)-ICMS11-WYT], the National Natural Science Foundation of China (NSFC, no. 81470170), and the Fundamental Research Funds for the Central Universities (lzujbky-2014-148). W.-A.Q. was supported by the Baskes Foundation and Robert H. Lurie Comprehensive Cancer Center at Northwestern University (Chicago, IL, USA).

\section{References}

1. Guthrie D and Gibson AL: Doxorubicin cardiotoxicity: Possible role of digoxin in its prevention. BMJ 2: 1447-1449, 1977.

2. Horwitz SB, Lothstein L, Manfredi JJ, Mellado W, Parness J, Roy SN, Schiff PB, Sorbara L and Zeheb R: Taxol: Mechanisms of action and resistance. Ann NY Acad Sci 466: 733-744, 1986.

3. Peterson DE and Sonis ST: Oral complications of cancer chemotherapy: Present status and future studies. Cancer Treat Rep 66 1251-1256, 1982

4. Erber R, Gluz O, Brünner N, Kreipe HH, Pelz E, Kates R, Bartels A, Huober J, Mohrmann S, Moustafa Z, et al: Predictive role of HER2/neu, topoisomerase-II- $\alpha$, and tissue inhibitor of metalloproteinases (TIMP-1) for response to adjuvant taxane-based chemotherapy in patients with intermediate-risk breast cancer: Results from the WSG-AGO EC-Doc trial. Breast Cancer Res Treat 150: 279-288, 2015.

5. Seely D, Wu P, Fritz H, Kennedy DA, Tsui T, Seely AJ and Mills E: Melatonin as adjuvant cancer care with and without chemotherapy: A systematic review and meta-analysis of randomized trials. Integr Cancer Ther 11: 293-303, 2012.

6. Lecumberri E, Dupertuis YM, Miralbell R and Pichard C: Green tea polyphenol epigallocatechin-3-gallate (EGCG) as adjuvant in cancer therapy. Clin Nutr 32: 894-903, 2013.

7. Qi F, Li A, Inagaki Y, Gao J, Li J, Kokudo N, Li XK and Tang W: Chinese herbal medicines as adjuvant treatment during chemo- or radio-therapy for cancer. Biosci Trends 4: 297-307, 2010.

8. Wang CY, Bai XY and Wang CH: Traditional Chinese medicine: A treasured natural resource of anticancer drug research and development. Am J Chin Med 42: 543-559, 2014.

9. Chatterjee K, Zhang J, Honbo N and Karliner JS: Doxorubicin cardiomyopathy. Cardiology 115: 155-162, 2010.

10. Bandyopadhyay A, Wang L, Agyin J, Tang Y, Lin S, Yeh IT, De K and Sun LZ: Doxorubicin in combination with a small TGF $\beta$ inhibitor: A potential novel therapy for metastatic breast cancer in mouse models. PLoS One 5: e10365, 2010.

11. Wang S, Wang L, Chen M and Wang Y: Gambogic acid sensitizes resistant breast cancer cells to doxorubicin through inhibiting P-glycoprotein and suppressing survivin expression. Chem Biol Interact 235: 76-84, 2015.

12. Thandavarayan RA, Giridharan VV, Arumugam S, Suzuki K, Ko KM, Krishnamurthy $\mathrm{P}$, Watanabe $\mathrm{K}$ and Konishi $\mathrm{T}$ : Schisandrin B prevents doxorubicin induced cardiac dysfunction by modulation of DNA damage, oxidative stress and inflammation through inhibition of MAPK/p53 signaling. PLoS One 10 e0119214, 2015.

13. Ashraf K, Mujeeb M, Ahmad A, Ahmad N and Amir M: Determination of curcuminoids in Curcuma longa Linn. by UPLC/Q-TOF-MS: An application in turmeric cultivation. J Chromatogr Sci 53: 1346-1352, 2015.

14. Yang FQ, Wang YT and Li SP: Simultaneous determination of 11 characteristic components in three species of Curcuma rhizomes using pressurized liquid extraction and high-performance liquid chromatography. J Chromatogr A 1134: 226-231, 2006.

15. Ray S, Chattopadhyay N, Mitra A, Siddiqi M and Chatterjee A: Curcumin exhibits antimetastatic properties by modulating integrin receptors, collagenase activity, and expression of $\mathrm{Nm} 23$ and E-cadherin. J Environ Pathol Toxicol Oncol 22: 49-58, 2003.

16. Leu TH, Su SL, Chuang YC and Maa MC: Direct inhibitory effect of curcumin on Src and focal adhesion kinase activity. Biochem Pharmacol 66: 2323-2331, 2003.
17. Lin ML, Lu YC, Chen HY, Lee CC, Chung JG and Chen SS: Suppressing the formation of lipid raft-associated Rac1/PI3K/Akt signaling complexes by curcumin inhibits SDF- $1 \alpha$-induced invasion of human esophageal carcinoma cells. Mol Carcinog 53: 360-379, 2014

18. Chen WC, Lai YA, Lin YC, Ma JW, Huang LF, Yang NS, Ho CT, Kuo SC and Way TD: Curcumin suppresses doxorubicin-induced epithelial-mesenchymal transition via the inhibition of TGF- $\beta$ and PI3K/AKT signaling pathways in triple-negative breast cancer cells. J Agric Food Chem 61: 11817-11824, 2013.

19. Hong JH, Lee G and Choi HY: Effect of curcumin on the interaction between androgen receptor and Wnt/ $\beta$-catenin in LNCaP xenografts. Korean J Urol 56: 656-665, 2015.

20. Zhang $X$, Zhang Y and Li Y: $\beta$-elemene decreases cell invasion by upregulating E-cadherin expression in MCF-7 human breast cancer cells. Oncol Rep 30: 745-750, 2013.

21. Shi H, Liu L, Liu LM, Geng J and Chen L: Inhibition of tumor growth by $\beta$-elemene through downregulation of the expression of UPA, UPAR, MMP-2, and MMP-9 in a murine intraocular melanoma model. Melanoma Res 25: 15-21, 2015.

22. Zhong Z, Dang Y, Yuan X, Guo W, Li Y, Tan W, Cui J, Lu J, Zhang Q, Chen X, et al: Furanodiene, a natural product, inhibits breast cancer growth both in vitro and in vivo. Cell Physiol Biochem 30: 778-790, 2012.

23. Zhong ZF, Hoi PM, Wu GS, Xu ZT, Tan W, Chen XP, Cui L, $\mathrm{Wu} \mathrm{T}$ and Wang YT: Anti-angiogenic effect of furanodiene on HUVECs in vitro and on zebrafish in vivo. J Ethnopharmacol 141: 721-727, 2012.

24. Zhong Z, Tan W, Chen X and Wang Y: Furanodiene, a natural small molecule suppresses metastatic breast cancer cell migration and invasion in vitro. Eur J Pharmacol 737: 1-10, 2014.

25. Zhong ZF, Li YB, Wang SP, Tan W, Chen XP, Chen MW and Wang YT: Furanodiene enhances tamoxifen-induced growth inhibitory activity of ERa-positive breast cancer cells in a PPAR $\gamma$ independent manner. J Cell Biochem 113: 2643-2651, 2012.

26. Li T, Xu XH, Tang ZH, Wang YF, Leung CH, Ma DL, Chen XP, Wang YT, Chen Y and Lu JJ: Platycodin D induces apoptosis and triggers ERK- and JNK-mediated autophagy in human hepatocellular carcinoma BEL-7402 cells. Acta Pharmacol Sin 36: 1503-1513, 2015

27. Zhong Z, Liu LJ, Dong ZQ, Lu L, Wang M, Leung CH, Ma DL and Wang Y: Structure-based discovery of an immunomodulatory inhibitor of TLR1-TLR2 heterodimerization from a natural product-like database. Chem Commun 51: 11178-11181, 2015

28. Gao JL, Shi JM, He K, Zhang QW, Li SP, Lee SM and Wang YT: Yanhusuo extract inhibits metastasis of breast cancer cells by modulating mitogen-activated protein kinase signaling pathways. Oncol Rep 20: 819-824, 2008.

29. Saraswati S, Alhaider AA and Agrawal SS: Punarnavine, an alkaloid from Boerhaavia diffusa exhibits anti-angiogenic activity via downregulation of VEGF in vitro and in vivo. Chem Biol Interact 206: 204-213, 2013.

30. Miltenburg NC and Boogerd W: Chemotherapy-induced neuropathy: A comprehensive survey. Cancer Treat Rev 40: 872-882, 2014.

31. Paus R, Haslam IS, Sharov AA and Botchkarev VA: Pathobiology of chemotherapy-induced hair loss. Lancet Oncol 14: e50-e59, 2013.

32. Wardill HR, Bowen JM and Gibson RJ: Chemotherapy-induced gut toxicity: Are alterations to intestinal tight junctions pivotal? Cancer Chemother Pharmacol 70: 627-635, 2012.

33. Daenen LG, Roodhart JM, van Amersfoort M, Dehnad M, Roessingh W, Ulfman LH, Derksen PW and Voest EE: Chemotherapy enhances metastasis formation via VEGFR-1-expressing endothelial cells. Cancer Res 71: 6976-6985, 2011.

34. Ran S: The role of TLR4 in chemotherapy-driven metastasis. Cancer Res 75: 2405-2410, 2015.

35. Park SI, Liao J, Berry JE, Li X, Koh AJ, Michalski ME, Eber MR, Soki FN, Sadler D, Sud S, et al: Cyclophosphamide creates a receptive microenvironment for prostate cancer skeletal metastasis. Cancer Res 72: 2522-2532, 2012.

36. Vyas D, Laput G and Vyas AK: Chemotherapy-enhanced inflammation may lead to the failure of therapy and metastasis. Onco Targets Ther 7: 1015-1023, 2014.

37. Xiong W, Ren ZG, Qiu SJ, Sun HC, Wang L, Liu BB, Li QS, Zhang W,Zhu XD, Liu L, et al: Residual hepatocellular carcinoma after oxaliplatin treatment has increased metastatic potential in a nude mouse model and is attenuated by Songyou Yin. BMC Cancer 10: 219, 2010. 
38. Biswas S, Guix M, Rinehart C, Dugger TC, Chytil A, Moses HL, Freeman ML and Arteaga CL: Inhibition of TGF- $\beta$ with neutralizing antibodies prevents radiation-induced acceleration of metastatic cancer progression. J Clin Invest 117: 1305-1313, 2007.

39. Pichot CS, Hartig SM, Xia L, Arvanitis C, Monisvais D, Lee FY, Frost JA and Corey SJ: Dasatinib synergizes with doxorubicin to block growth, migration, and invasion of breast cancer cells. Br J Cancer 101: 38-47, 2009.

40. Kim S, Harris M and Varner JA: Regulation of integrin $\alpha$ $\mathrm{v} \beta 3$-mediated endothelial cell migration and angiogenesis by integrin $\alpha 5 \beta 1$ and protein kinase A. J Biol Chem 275 : 33920-33928, 2000

41. Parsons JT, Martin KH, Slack JK, Taylor JM and Weed SA Focal adhesion kinase: A regulator of focal adhesion dynamics and cell movement. Oncogene 19: 5606-5613, 2000.

42. Chen HC, Appeddu PA, Isoda H and Guan JL: Phosphorylation of tyrosine 397 in focal adhesion kinase is required for binding phosphatidylinositol 3-kinase. J Biol Chem 271: 26329-26334, 1996.

43. Webb DJ, Donais K, Whitmore LA, Thomas SM, Turner CE, Parsons JT and Horwitz AF: FAK-Src signalling through paxillin, ERK and MLCK regulates adhesion disassembly. Nat Cell Biol 6: 154-161, 2004.

44. Thant AA, Nawa A, Kikkawa F, Ichigotani Y, Zhang Y, Sein TT, Amin AR and Hamaguchi M: Fibronectin activates matrix metalloproteinase-9 secretion via the MEK1-MAPK and the PI3K-Akt pathways in ovarian cancer cells. Clin Exp Metastasis 18: 423-428, 2000

45. Woodfield RJ, Hodgkin MN, Akhtar N, Morse MA, Fuller KJ, Saqib K, Thompson NT and Wakelam MJ: The p85 subunit of phosphoinositide 3 -kinase is associated with $\beta$-catenin in the cadherin-based adhesion complex. Biochem J 360: 335-344, 2001.
46. Vu TH, Shipley JM, Bergers G, Berger JE, Helms JA, Hanahan D, Shapiro SD, Senior RM and Werb Z: MMP-9/gelatinase B is a key regulator of growth plate angiogenesis and apoptosis of hypertrophic chondrocytes. Cell 93: 411-422, 1998.

47. Bartsch JE, Staren ED and Appert HE: Matrix metalloproteinase expression in breast cancer. J Surg Res 110: 383-392, 2003.

48. Van Keymeulen A, Wong K, Knight ZA, Govaerts C, Hahn KM, Shokat KM and Bourne HR: To stabilize neutrophil polarity, PIP3 and Cdc42 augment RhoA activity at the back as well as signals at the front. J Cell Biol 174: 437-445, 2006.

49. Nobes CD and Hall A: Rho, rac and cdc42 GTPases: Regulators of actin structures, cell adhesion and motility. Biochem Soc Trans 23: 456-459, 1995.

50. Carlier MF, Ducruix A and Pantaloni D: Signalling to actin: The Cdc42-N-WASP-Arp2/3 connection. Chem Biol 6: R235-R240, 1999.

51. Rohatgi R, Ma L, Miki H, Lopez M, Kirchhausen T, Takenawa T and Kirschner MW: The interaction between N-WASP and the Arp $2 / 3$ complex links Cdc42-dependent signals to actin assembly. Cell 97: 221-231, 1999.

52. Yamazaki D, Kurisu S and Takenawa T: Regulation of cancer cell motility through actin reorganization. Cancer Sci 96: 379-386, 2005.

53. Westermann S and Weber K: Post-translational modifications regulate microtubule function. Nat Rev Mol Cell Biol 4: 938-947, 2003. 